\title{
A novel protocol for generating intact, whole-head spider cephalothorax tissue sections
}

\author{
Skye M Long ${ }^{1}$ \\ 'Biology Department, University Massachusetts Amherst, Amherst, MA 01003, USA
}

BioTechniques 64:163-169 (April 2018) 10.2144/btn-2018-2002

Keywords: arthropod morphology; fluorescent microscopy; spider morphology; whole head sectioning

The diversity of spider behavior and sensory systems provides an excellent opportunity for comparative studies of the relationship between the brain and behavior. However, the morphology of spiders poses a challenge for histologists since the spider cephalothorax contains heterogeneous tissues and has both tough external and internal sclerotized regions. Unlike the heads of insects, the cephalothorax is highly pressurized, which can cause tissues to shift during processing and can reduce tissue cohesion in thick sections. This work describes a novel protocol for producing thick whole-head sections for morphological study by softening the exoskeleton and stabilizing friable tissue, without freezing or dehydration. It also presents an effective wholehead Dil staining method that uses minimal dehydration and highlights neural structures.

The enormous diversity of arthropod behavior and sensory system morphology provides an ample resource for comparative studies of the relationship between the brain and behavior. In insects, neuroanatomical studies have illustrated the relationship between brain morphology and behavioral complexity [1]. For example, the mushroom bodies in the central nervous systems of specialist dung-feeding scarab beetle species (Coleoptera: Scarabaeidae) were smaller and comprised of fewer neurons than generalist plant-feeding scarab beetle species [2].

Additionally, brain morphology can be used to explore cladistic relationships. Large-scale analyses using neuroanatomical characteristics have helped clarify the relationships between insects, crustaceans, onychophorans, and chelicerates [3,4]. Results from studies of arthropod brains can even be compared with mammalian systems as there are convergent commonalities, such as increased compartmentalization, in how vertebrate and invertebrate brains evolve in response to behavioral complexity [5].
Spiders offer a rich behavioral repertoire, and with their sensory system diversity provide a different evolutionary pathway for studying the evolution of arthropod neuromorphology. While the insect's brain is dominated by both olfactory and visual stimuli, the spider's brain has no antennal lobe and much of the protocerebrum is dedicated to visual input $[6,7]$. Compared to insects, little is known about neuromorphological variation within many spider families. The few studies that have examined spider brains have found some tantalizing differences across species [8]. Like scarab beetles, the relative volume of spider brain regions varied with different foraging behaviors $[1,8]$.

Like all study organisms, the morphology of the spider is unique and requires modifications when using traditional histochemical protocols. The spider's body is covered in a hard exoskeleton that must be removed or softened before sectioning. Additionally, the spider's cephalothorax, or prosoma, contains everything that is separated into the head and thoracic segments in insects. The muscles, fat, digestive tissue, sclerotized esophagus and mouthparts, sucking stomach, venom glands, brain, and optical tracks all share space in the cephalothorax, surrounded by pressurized hemolymph. Under normal pressure, these tissues remain in their respective locations, but compromising the cephalothorax, resulting in the loss of pressure, can cause these tissues to become displaced.

Sectioning tissue with an intact exoskeleton can cause several problems. The inflexible exoskeleton can splinter and damage paraffin and resin sections; if gelatin-agar blocks the pressure of the leading edge of the blade against the exoskeleton, it can pull the sample from the block or cause uneven sectioning. Removing the spider's brain from the cephalothorax can help limit problems. While removal of the spider's brain and associated visual centers is possible, it takes a practiced hand because the brain is surrounded by robust leg muscles, digestive diverticula, venom glands, the sucking stomach, and fatty tissue [9]. Dissection can easily result in trauma to brain tissue or the loss of delicate optical tracts, especially in smaller animals. Additionally, the sclerotized esophagus, which bisects the brain and is

\section{METHOD SUMMARY}

By softening the exoskeleton and embedding the tissue in a gelatin/agar matrix to retain the position of internal structures, thick sections of whole spider cephalothorax tissues appropriate for fluorescent confocal microscopy can be prepared. Additionally, a modified whole-head Dil staining technique for imaging nervous tissue is described. 
flanged on both the anterior and posterior end, must be removed to prevent it from tearing the tissue during sectioning, which often severely damages brain tissue.

With practice and modification, traditional paraffin and resin embedding techniques can solve many of these problems and can produce very nice results. The benefit of the current method is the ease of use, the use of relatively non-toxic chemicals, and the ability to produce thick sections that can be stained with lipophilic dyes. This study describes a simple process that yields thick sections of whole cephalothorax tissues appropriate for fluorescent confocal microscopy. In this method, I first soften the exoskeleton and then embed the tissue in a gelatin/agar matrix to retain the position of internal structures. A modified whole-head Dil staining technique that allows for imaging nervous tissue is also presented.

\section{Materials \& methods}

\section{Study organisms}

Spiders were sampled from 20 different families initially selected to be part of a large comparative study: Agelenidae, Amaurobiidae, Antrodiaetidae, Araneidae, Ctenidae, Deinopidae, Filistatidae, Hypochilidae, Lycosidae, Miturgidae, Nephilinae, Oxyopidae, Philodromidae, Pholcidae, Pisauridae, Salticidae, Scytodidae, Theridiidae, Thomisidae and Uloboridae [10]. Spiders ranged from 3 to $21 \mathrm{~mm}$ in length and were collected from fields in western Massachusetts, USA; Archbold Biological Station, FL, USA; or Jacksonville, FL, USA from 2012-2014. Antrodiaetidae were purchased from BugsInCyberspace.com, OR, USA. Prior to dissection, spiders were housed in $18 \times 13 \times 10 \mathrm{~cm}$ clear plastic cages, were fed crickets (Acheta domesticus) weekly, and had constant access to water. Spiders were typically housed for less than 2 weeks prior to dissection.

\section{Dissection \& fixation}

Spiders were anesthetized by placing them in the freezer for approximately 5-20 min depending on body size, taking care not to allow the spider to freeze during this step. The opisthosoma, legs, the tips of the chelicerae, and external mouthparts were removed by microdissection in chilled $0.1 \mathrm{M}$ 7.4pH PBS using a pair of small iris scissors. Care was taken not to pierce the cephalothorax prior to fixation to avoid hemolymph leakage and shifting of internal anatomy. Immediately following dissection, the cephalothorax was placed in 3\% paraformaldehyde/3\% glutaraldehyde at $\mathrm{pH} 7.4$ in $0.1 \mathrm{M}$ PBS buffer (Electron Microscopy Sciences, PA, USA) and fixed overnight at $4^{\circ} \mathrm{C}$. Fixed samples were rinsed three times using fresh 0.1M 7.4pH PBS under light agitation.

\section{Softening the exoskeleton}

To soften the exoskeleton, samples were then placed into individual Eppendorf tubes or scintillation vials filled with $3-5 \mathrm{ml}$ of freshly mixed 10\% Nair solution in PBS (Nair Shower Power Max, Church \& Dwight Co., Inc., NJ, USA; Table 1) and allowed to soften for 2-3 weeks at $4^{\circ} \mathrm{C}$. Other Nair solutions were not tried, but may work. Nair is the brand name of a common depilatory cream readily available commercially. Although the concentrations of the different components are unknown, it is likely the combination of the sodium hydroxide, calcium hydroxide, and urea that produce the softening effect. Sodium hydroxide has been used previously as a cuticle-softening agent in insects [11], and urea is used in the SCALE tissue clearing technique [12]. The Nair solution was changed weekly. The firmness of each exoskeleton was tested by applying light pressure to the top of the cephalothorax with a blunt instrument. If the cephalothorax deformed easily under pressure the sample was ready for embedding. If not, it was returned to the Nair solution for an additional week. Spiders with thinner cuticles, such as Pholcidae, were ready for sectioning after 2 weeks, while those with thicker cuticles, such as Salticidae, needed to soften for 3 weeks. The volume of Nair solution used varied with the size of the spider, but was always enough to completely cover the cephalothorax.

After softening, the Nair was rinsed from each cephalothorax using 0.1M 7.4pH PBS and then the cuticle gently brushed with a small micro-brush (EasyinSmile Dental, Staten Island, NY, USA) until all Nair was removed. It was very important to remove all the Nair from the external cuticle, as residual Nair could diffuse into the gelatin/ agar matrix and cause cracks in the block during sectioning. The agitation of the brush also seemed to further aid in softening the cuticle. After removing the Nair from the surface of the cuticle, the cephalothorax was rinsed by soaking it in fresh $0.1 \mathrm{M} 7.4 \mathrm{pH}$ PBS three times for 10 minutes each.

\section{Embedding \& Sectioning}

To prepare the cephalothorax for embedding, iris scissors were used to remove the sternum by carefully cutting the pieces of chitin securing it to the leg openings. The sternum was then gently peeled away from the spider using fine forceps. Next, several perforations were made in the dorsal side of the cuticle using a very fine insect minuten pin to allow the gelatin/agar embedding medium to infiltrate the dorsal portion of the exoskeleton and to help the medium adhere to the cuticle. Larger spiders with a thicker cuticle required more perforations than did those with a thinner cuticle. Perforations were concentrated around the eyes to ensure that the gelatin/agar mixture saturated the retina and fine optic nerves located behind the cornea.

Perforated cephalothoraxes were placed into individual Eppendorf tubes filled with

Table 1. Solutions.

Solution name Directions

$10 \%$ Nair solution

1. Mix 10\% Nair Shower Power Max with fresh 0.1M 7.4pH PBS using a magnetic stir bar on high speed until the consistency of thin yogurt. 2. Use fresh. Do not store.

Gelatin/agar solution

1. Mix 20 g gelatin (300 Bloom/Porcine) with $10 \mathrm{~g}$ low melting point agar in $250 \mathrm{ml}$ round media storage bottle. Add $0.1 \mathrm{M} 7.4 \mathrm{pH}$ PBS to $200 \mathrm{ml}$. 2 . Place lid on bottle and shake until mixed. Allow to sit at room temp for 15 minutes. 3. Place in $60-65^{\circ} \mathrm{C}$ water bath until melted. Make the night before embedding. Swirl to mix before use. Can be stored at $4^{\circ} \mathrm{C}$ in a closed container for several weeks. Do not use if frozen or if left open.

Dil staining solution

1. Mix enough Dil crystals into $100 \%$ ETOH to make a medium pink solution, approximately $5 \%$. If too much Dil is added, there is visible precipitate in the solution, add more $100 \% \mathrm{ETOH}$. 2. Dilute the $100 \%$ Dil/ETOH mixture to $70 \%$ using $\mathrm{dH}_{2} 0$.Can be stored in the dark at $4{ }^{\circ} \mathrm{C}$. 3 . Dil can be recovered from the $100 \%$ or $70 \%$ solution by allowing the ETOH to evaporate. 
ADVERT 


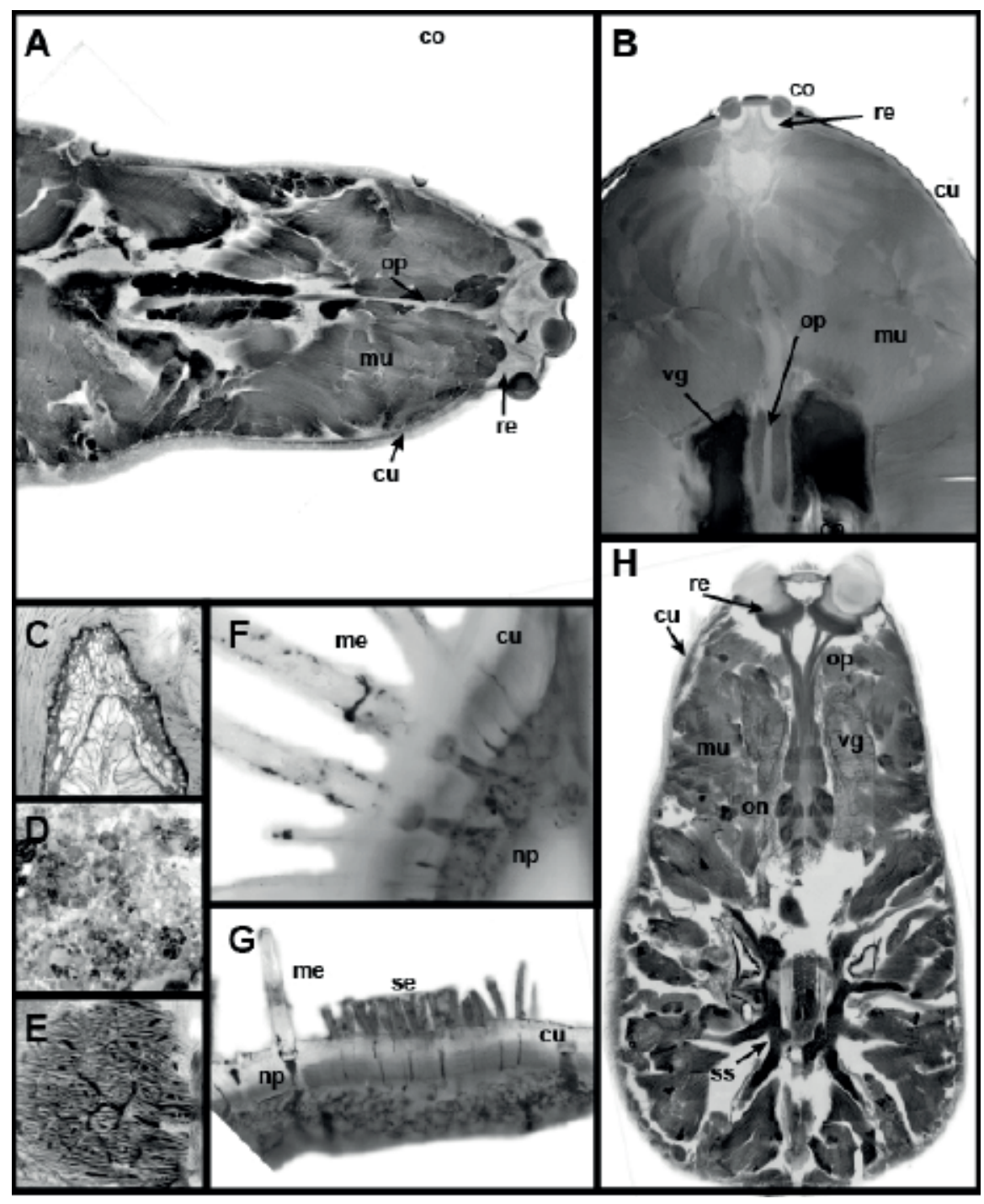

Figure 1. Examples of whole-head sectioned tissues. Images have been inverted for clarity. All images are from $100 \mu \mathrm{m}$ sections prepared with the described cuticle softening and embedding protocol. Images A \& B are not Dil stained. Images C-H are Dil Stained. Images A, B, F, and G are conventional fluorescent images. Images $\mathrm{C}$ - $\mathrm{E}$ and $\mathrm{H}$ are confocal images. (A) A 10x image of Agelenopsis pennsylvanica. (B) A 10x image of a $100 \mu \mathrm{m}$ section of Peucetia viridans. (C) A 20x image of a Phidippus audax venom gland. (D) A 20x image of fat bodies in $P$. audax. (E) A 20x image of muscle tissue in $P$. audax. (F) A 20x image of mechanosensory sensilla and connecting dendrites in the cuticle of $A$. pennsylvanica. (G) A 20x image of sensory sensilla and connecting dendrites in the cuticle of Rabidosa rabida. (H) A 10x fluorescent image of $R$. rabida.

co: Cornea; cu: Cuticle; me: Mechanosensory sensillum; mu: Muscle; np: Nerve projection; on: Optic neuropil; op: Optic nerve; re: Retina; se: Sensory hairs; ss: Sucking stomach; vg: Venom gland.

3-5 ml of warm, pre-made gelatin/agar embedding solution (Table 1, similar to Jones \& Calabresi 2007). Cephalothoraxes soaked overnight in a $60^{\circ} \mathrm{C}$ water bath. Using $5 \mathrm{ml}$ micro-beakers (Fisher Scientific, PA, USA) as embedding molds, each cephalothorax was immersed in fresh gelatin/agar embedding solution and allowed to harden overnight at $4^{\circ} \mathrm{C}$. Cephalothoraxes were embedded dorsal side down, which left excess embedding material at the ventral edge of the sample for attaching to the vibratome chuck.
Excess embedding media was trimmed from the sample blocks immediately after removal from the $4^{\circ} \mathrm{C}$ refrigerator. If samples became too warm, the embedding media could separate from the exoskeleton. In this case, samples were returned to the refrigerator for an hour before re-trimming. It was found that if samples accidently got wet from condensation on the outside of the mold at this stage, the gelatin within the media began to melt, causing the sample to pull away from the media. The embedding media was trimmed, leaving 1-mm excess of media on all sides, except the ventral edge of the cephalothorax, where $2 \mathrm{~mm}$ was left for attachment to the vibratome chuck. Leaving more than $2 \mathrm{~mm}$ on the ventral edge made the sample unstable during sectioning and led to uneven sections and sometimes separation of the sample from the embedding media. After trimming, the gelatin/agar embedded samples were placed into 3\% paraformaldehyde/3\% glutaraldehyde at pH 7.4 in 0.1M PBS buffer and allowed to post-fix overnight at $4^{\circ} \mathrm{C}$. For very large samples (over $1 \mathrm{~cm}$ ), the sample was allowed to post-fix for up to $24 \mathrm{~h}$.

After post-fixation, samples were rinsed three times for 10 min each in fresh washes of 0.1M 7.4pH PBS. Samples were either sectioned immediately, or stored in a $20 \%$ weight/volume aqueous sucrose solution for up to 2 weeks. Transverse sections were cut using a 1000 Plus Sectioning System vibratome (The Vibratome Co., MO, USA) in $4^{\circ} \mathrm{C} 0.1 \mathrm{M} 7.4 \mathrm{pH}$ PBS. Samples were attached to the chuck by the ventral edge with superglue (Krazy Glue, OH, USA) and allowed to dry. Samples were aligned in the vibratome with the anterior-posterior axis parallel to the blade's edge. Sectioning with the blade passing along the anteriorposterior axis placed too much blade pressure on the leading edge of the sample, causing uneven or torn sections. 100- $\mu \mathrm{m}$ sections were cut with a double-bladed safety blade with the vibratome set to a high amplitude and slow speed. Sections were placed into 0.1M 7.4pH PBS and transferred to free-floating section storage solution (FD Neurotechnologies, MD, USA) for storage at $-20^{\circ} \mathrm{C}$.

\section{Dil staining}

Before imaging, sections were rinsed for 10 minutes three times in 0.1M 7.4pH PBS at room temperature. Sections were stained with a modified whole-head Dil protocol [14] to enhance neural structures. For staining, sections were immersed in 50\% ethanol for $5 \mathrm{~min}, 70 \%$ Dil/ethanol solution (Table 1) for $5 \mathrm{~min}, 70 \%$ ethanol for $5 \mathrm{~min}, 50 \%$ ethanol for $5 \mathrm{~min}$, and $1 \times$ PBS for $10 \mathrm{~min}$, all under gentle agitation at room temperature and protected from light with aluminum foil. Dil is a lipophilic stain that requires an intact cellular membrane. The minimal dehydration prevented the degradation the neuronal cellular membranes. In addition, dehydration can also cause tissue artifacts in other fatty tissues that can lead to warping (Figure 3). Sections were kept in dark conditions in a $20 \%$ weight/volume sucrose solution for 


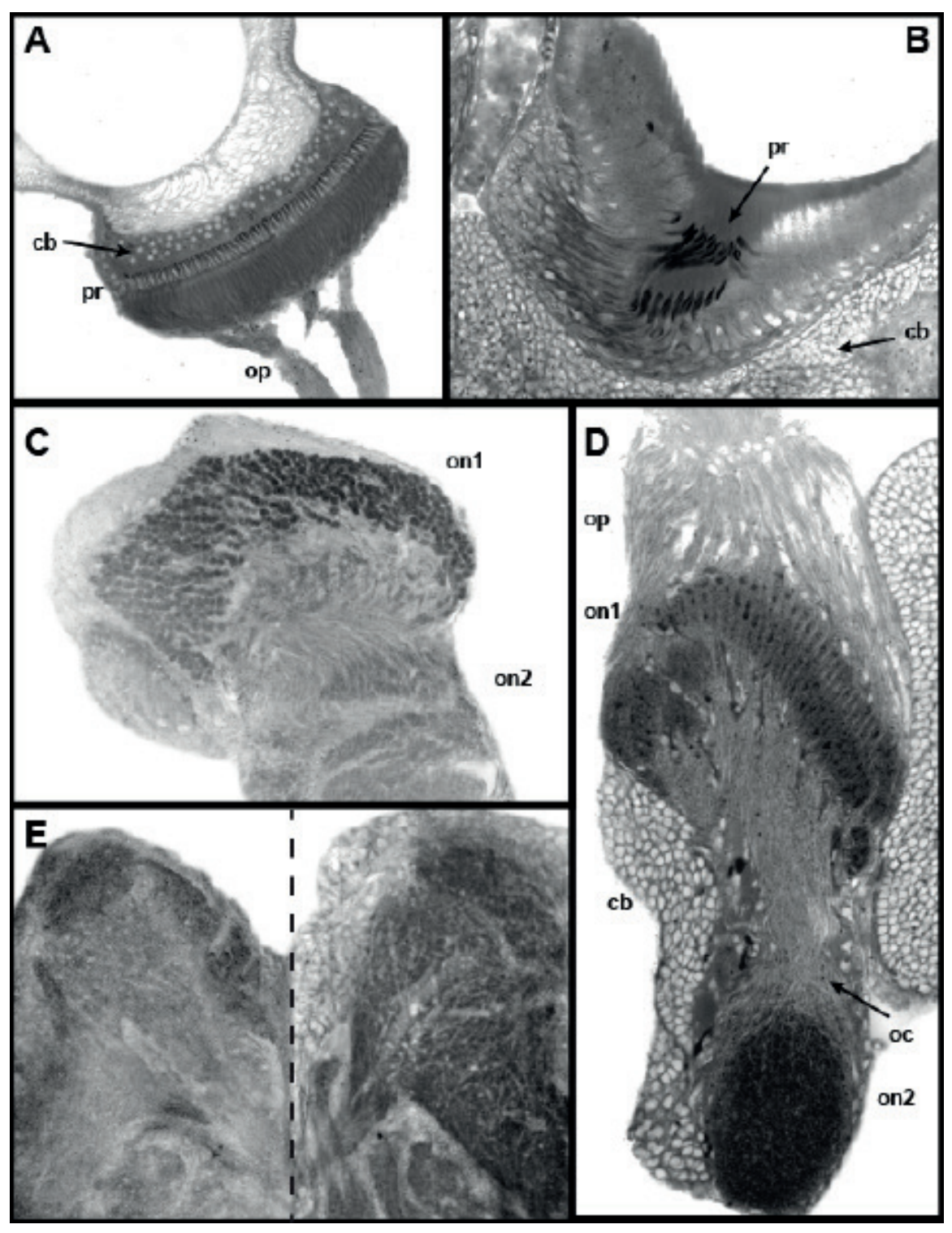

Figure 2. Examples of Dil stained tissue. All images represent Dil-stained samples except for the left panel of E, which was taken prior to Dil staining. All panels are 20x confocal images from $100 \mu \mathrm{m} \mathrm{sec-}$ tions prepared using the protocol described in this article. (A) Posterior medial eye retina of $R$. rabida. (B) Principal eye retina of $P$. audax. (C) First and second optic neuropils of the secondary eyes from $P$. audax. (D) First and second optic neuropils of the principal eye from $P$. audax. (E) Left panel: left mushroom body head of $R$. rabida imaged prior to Dil staining. Right panel: The right mushroom body head from the same section after Dil staining.

cb: Cell bodies; oc: Optic chiasma; on1: First optic neuropil; on2: Second optic neuropil; op: Optic nerve; pr: Photoreceptors.

\section{$3-5$ days at $4^{\circ} \mathrm{C}$ to allow the stain to perfuse} the sample.

\section{Imaging}

Prior to confocal imaging, sections were again rinsed for 10 minutes three times in 0.1M 7.4pH PBS. Tissue slices were reversibly mounted between two plates of \#1-1/2 coverslip glass (Fisher Scientific, PA, USA) using Fluoro-Gel III mounting solution containing DAPI (Electron Microscopy Sciences, PA, USA), which counterstained the nuclei. The sandwiched sample was secured to a Fisher Superfrost Plus slide with tape and imaged on a Nikon Eclipse Ti confocal microscope, using NIS-Elements AR acquisition software. After one side was imaged, the tape holding the sandwiched section was detached from the slide, the cover slipped sample was flipped, re-attached to the slide and imaged. After imaging, the sandwiched section was soaked in $0.1 \mathrm{M} \mathrm{7.4pH}$ PBS to remove the tissue from cover slips, and the section was then placed back into the free-floating storage solution and returned to $-20^{\circ} \mathrm{C}$.

This method was followed for conventional fluorescent imaging with the only exception being the type of microscope used. For conventional fluorescent imaging (Figure 1A, B, F \& G), a Zeiss Axiolmager Z2 with Apotome controlled with AxioVision v. 4.8 was used. For confocal and conventional fluorescent Dil stained images (Figure 1C-H and Figure 2), $488 \mathrm{~nm}$ laser was used. For non-Dil-stained images (Figure 1A, B, and Figure 2E, left panel), a $561 \mathrm{~nm}$ laser was used.

The images were exported from the microscopy software and imported into Photoshop CC 2017.0.0 as.tif files. The images were inverted for clarity, and an exposure adjustment layer was used to enhance contrast.

\section{Resin-embedded sections}

For comparison to the method described, three adult Hogna Carolinensis were processed as follows. Animals were dissected and fixed as described above. After fixation, samples were dehydrated to 100\% ethanol. Samples were then prepared for spurs embedding. Samples were placed in $100 \%$ acetone for 10 minutes, then $50 \%$ acetone:50\% spurs overnight, then 10\% acetone:90\% spurs for 8 hours, then $100 \%$ spurs for 8 hours or overnight. All steps were done on a shaker at room temperature. Samples were then embedded in silicone molds and polymerized in the oven for 12 hours at $70^{\circ} \mathrm{C}$. Once cooled, samples were sectioned at $40 \mu \mathrm{m}$ and placed on Fisher Superfrost Plus slides and dried overnight on a slide warmer.

Dried slides were stained with $1 \%$ methylene blue and a coverslip was mounted using CytoSeal mounting medium (Electron Microscopy Sciences, PA, USA). Sections were imaged at 10x using a Zeiss Axiolmager Z2 with Apotome controlled with AxioVision v. 4.8. The images were exported from the microscopy software and imported into Photoshop CC 2017.0.0 as .tif files, and an exposure adjustment layer was used to enhance contrast.

\section{Results \& discussion}

Samples that were softened prior to embedding sectioned cleanly, without tearing or pulling from the embedding material. Spider tissue processed with this method was easy to section and image with little apparent damage. Problems associated with muscles pulling away from exoskeleton, torn or lost sections and tissue, and loss of delicate structures were virtually 


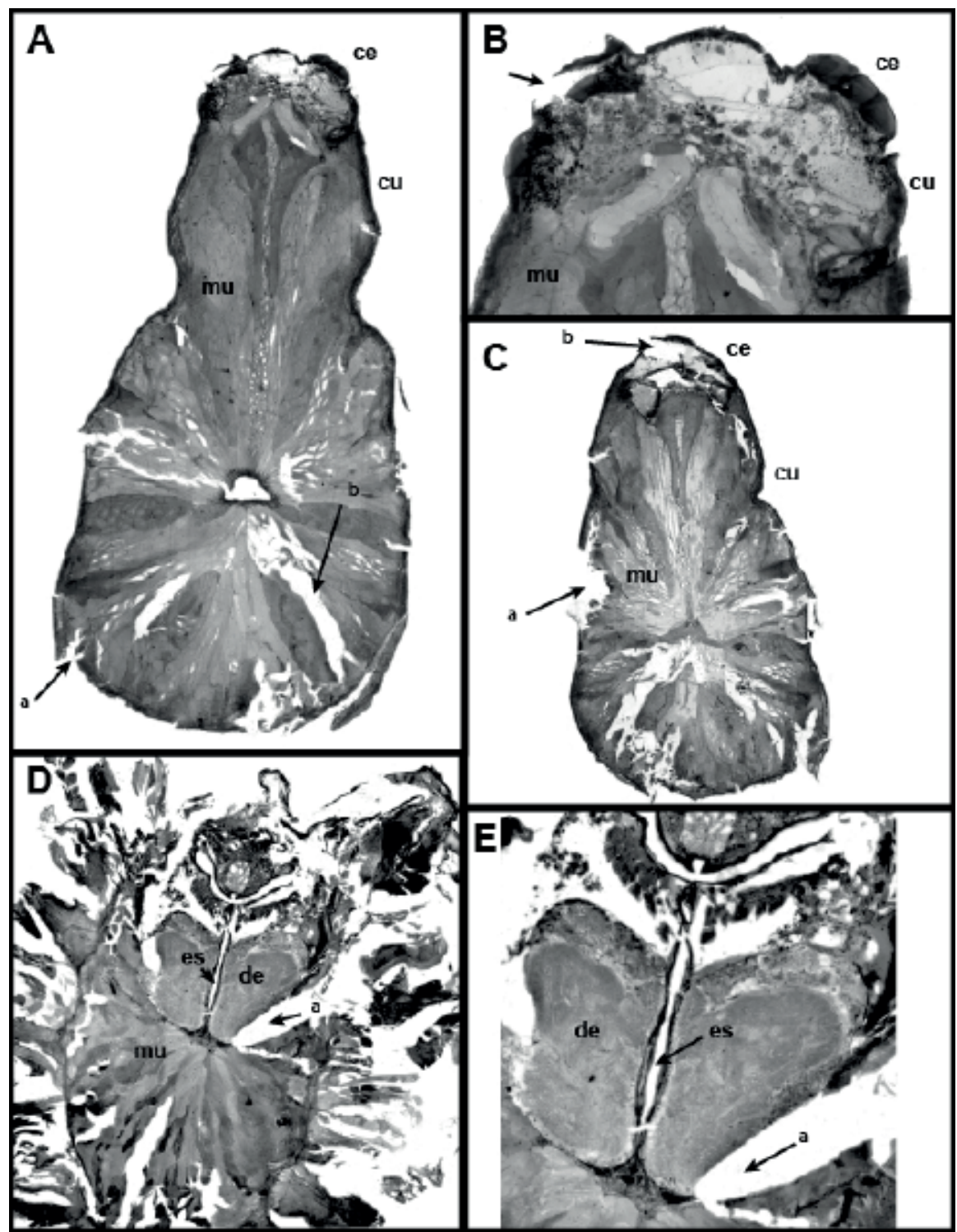

Figure 3. Examples of resin-embedded, methylene blue-stained samples. All panels are 10x light microscopy images from $40 \mu \mathrm{m}$ sections prepared using the resin protocol described in this article. All images are from adult Hogna carolinensis. (A) Dorsal section through cephelothorax. The arrow (a) indicates a disruption of the cuticle that resulted in tearing of the muscle tissue. Arrow (b) indicates area of muscle tissue loss. (B) Close-up of retinal region of A. The arrow indicates tearing of the cornea. (C) A dorsal section showing multiple disruptions and tissue loss. Arrow (a) indicates a missing region of the cuticle and tissue. Arrow (b) indicates disruption of the cuticle and missing tissue. (D) Section showing the ventral region. This region does not have enclosing cuticle. Arrow (a) shows an area of deformed tissue, possibly caused by shrinkage during dehydration. (E) Close-up of D.

ce: Cuticle of the eye; cu: Cuticle; de: Deutocerebrum; es: Esophagus; mu: Muscle.

absent (Figure 1 compared to Figure 3). Dil staining provided a method to observe all internal nervous structures (Figure 2). The contrast of nervous structures in Dil-stained tissues (Figure 2E, right panel) improved over background fluorescence provided by glutaraldehyde (Figure 2E, left panel). Fluorescence from the Dil also bled over into the $561 \mathrm{~nm}$ channel, giving it higher contrast than was seen before Dil staining.

Because Nair contains calcium hydroxide and sodium hydroxide, which breaks down the integrity of the cephalo- thorax, it is important to take the samples out of the cream once a week to check that the cephalothorax has not been significantly weakened. Samples were stored in Nair for up to 4 weeks without seeing any cephalothoraxes that were completely dissolved, but it is possible that this could occur with long term storage. In addition, the Nair solution tends to remove pigment from the cephalothorax, which would preclude it from use in studies where preserving the natural color of the cephalothorax is required. Finally, because this is a commercial product, the researcher has no control or knowledge of the concentrations of chemicals it contains or control over changes in formulation.

Prior attempts at whole-head sectioning with traditional resin techniques (Figure 3) and various versions of the current method revealed fractures of the exoskeleton and tearing, loss or deformation of tissues (Figure 3). Trials with gelatin/agar embedding, minus the infiltration step and secondary fixation, resulted in the entire cephalothorax pulling out of the gelatin/agar block during sectioning. This rendered the samples completely unusable. Samples processed with the secondary fixation, but not the infiltration step, did not pull out of the block, but internal tissues such as the muscle separated from the section and were lost, either on the vibratome blade or when floated in the $0.1 \mathrm{M} 7.4 \mathrm{pH}$ PBS solution. This disrupted the internal integrity of the sections and made images from them difficult to interpret.

With the current method, problems largely occurred in one of two ways. If the Nair step was too short, the cephalothorax tore, possibly resulting in tearing of the section. Additionally, if the secondary fixation step was too short, samples pulled out of the blocks or produced sections with uneven thicknesses due to the internal matrix being too soft. This was a problem in very large animals.

When prepared correctly, samples were robust and could withstand multiple rounds of imaging, opening up the possibility of comparative imaging techniques or multiple staining procedures on the same tissue sample. Additionally, the long storage time - up to 6 years at $-20^{\circ} \mathrm{C}$ (FD Neurotechnologies) - for free-floating sections allows researchers to return to samples to perform additional staining. Although agargelatin can interfere with some antigen sites, the elimination of a dehydration step, or the very limited dehydration for the Dil staining, reduces the loss of antigen reactivity and the need for antigen retrieval, while preserving tissue cytoarchitecture. With gelatin creating an internal matrix that is contained by the exoskeleton, the internal relationships of the different tissues were maintained without separating and spreading out, as can happen with spiders that have little internal integrity such as the lightly muscled Pholcidae.

It may be possible to pre-stain the sample prior to embedding with gelatinagar by soaking the perforated, sternum- 
removed samples in stain or antibodies, then following the embedding procedure as described here.

This method was used successfully on spiders ranging in body length from 3 to $21 \mathrm{~mm}$, and in sections ranging in thickness from 70 to $100 \mu \mathrm{m}$. This inexpensive and simple method for creating thick wholehead sections for spiders will improve the accessibility of these fascinating and little understood organisms, especially for studies involving brain or cephalothorax structures.

\section{Acknowledgements}

The author is grateful to Glen Marrs at Wake Forest University for his suggestion, advice and guidance for using Nair as a cuticle softening chemical and Cynthia Harley, Anthony Auletta, and Marc Seid for their advice on spider dissection techniques. The author would also like to thank Elizabeth Jakob for her help in editing this manuscript and Wulfi Gonenberg for his help with the figures. This work was supported by NSF (IOS 0952822 to Elizabeth Jakob).

\section{Competing \& financial interests disclosure}

This work was supported by NSF (IOS 0952822 to Elizabeth Jakob). The author has no other relevant affiliations or financial involvement with any organization or entity with a financial interest in or financial conflict with the subject matter or materials discussed in the manuscript apart from those disclosed.

No writing assistance was utilized in the production of this manuscript.

\section{References}

1. Strausfeld NJ. Arthropod Brains: Evolution, Functional Elegance, and Historical Significance. Harvard University Press (2012).

2. Farris SM. Structural, functional and developmental convergence of the insect mushroom bodies with higher brain centers of vertebrates. Brain. Behav. Evol. 72, 1-15 (2008).

3. Strausfeld NJ, Strausfeld CM, Loesel R, Rowell D, Stowe S. Arthropod phylogeny: onychophoran brain organization suggests an archaic relationship with a chelicerate stem lineage. Proc. Biol. Sci. 273, 1857-1866 (2006).

4. Strausfeld NJ. Brain organization and the origin of insects: an assessment. Proc. Biol. Sci. 276, 1929-1937 (2009).

5. Farris SM. Evolutionary convergence of higher brain centers spanning the protostome-deuterostome boundary. Brain. Behav. Evol. 72, 106122 (2008).
6. Damen WG, Hausdorf M, Seyfarth EA, Tautz D. A conserved mode of head segmentation in arthropods revealed by the expression pattern of Hox genes in a spider. Proc. Natl Acad. Sci. USA 95, 10665-10670 (1998).

7. Barth FG. A Spider's World: Senses and Behavior. Springer Science \& Business Media (2002).

8. Weltzien P, Barth FG. Volumetric measurements do not demonstrate that the spider brain 'central body' has a special role in web building. J. Morphol. 208, 91-98 (1991).

9. Foelix R. Biology of Spiders. Oxford University Press (2010).

10. Long S. Spider Brain Morphology \& Behavior. University of Massachusetts Amherst (2016).

11. Barbosa P, Berry DL, Kary CS. Problems of sclerotized chitin: softening insect cuticle. In: Insect Histology: Practical Labratory Techniques. John Wiley \& Sons, Chichester, UK, (2014).

12. Hama H, Kurokawa $\mathrm{H}$, Kawano $\mathrm{H}$ et al. Scale: a chemical approach for fluorescence imaging and reconstruction of transparent mouse brain. Nat. Neurosci. 14, 1481-1488 (2011).

13. Jones M, Calabresi P. Agar-gelatin for embedding tissues prior to paraffin processing. Biotechniques 42, 569-570 (2007).

14. Boyle DL, Takemoto LJ. Confocal microscopy of human lens membranes in aged normal and nuclear cataracts. Investig. Ophthalmol. Vis. Sci. 38, 2826-2832 (1997).

Received: 14 February 2017; Accepted for publication: 2 March 2018

Address correspondence to Skye M Long, Biology Department, University Massachusetts Amherst, Amherst, MA 01003, USA.

E-mail: smlong@umass.edu

To purchase reprints of this article, contact s.cavana@future-science.com

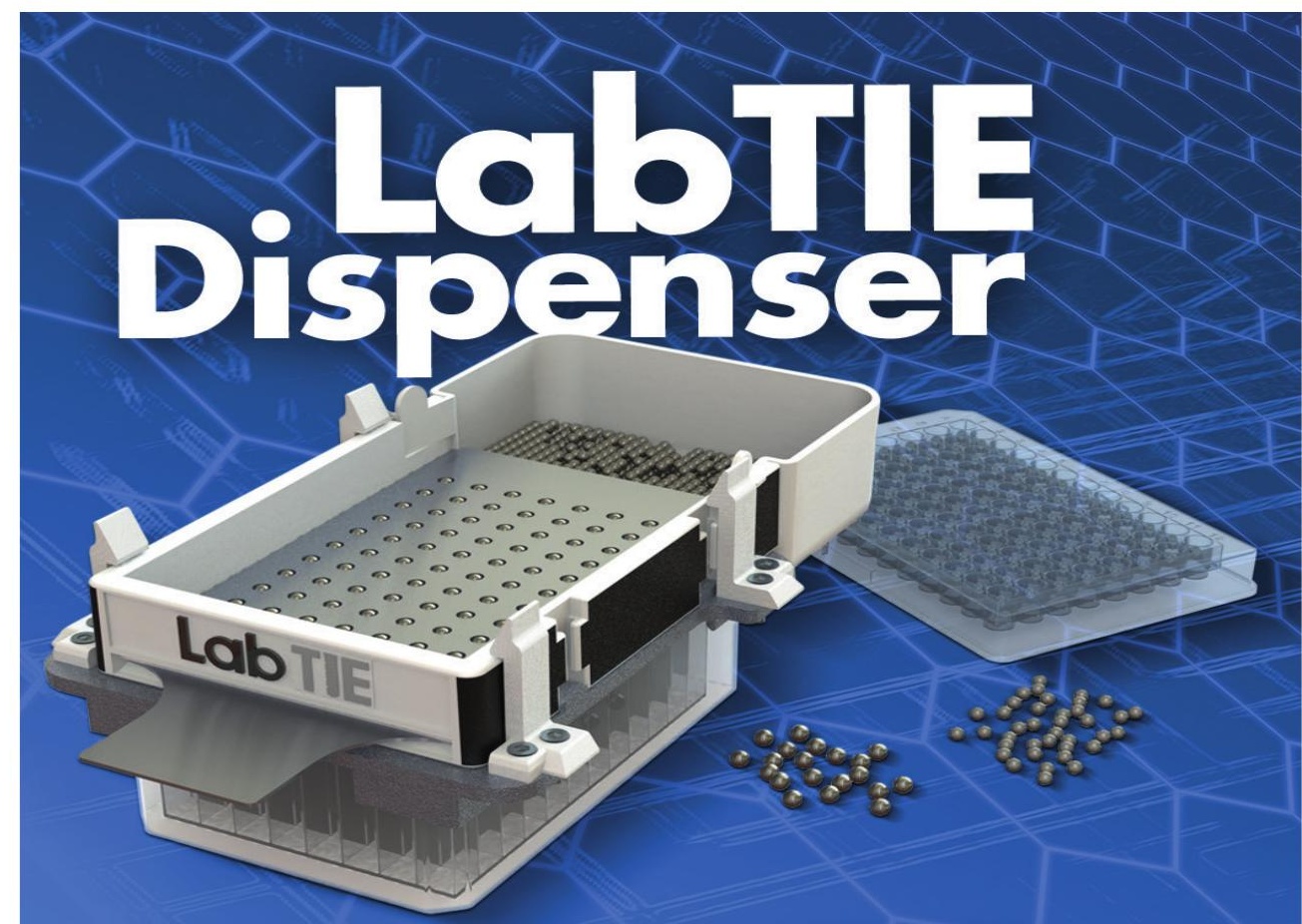

- EASILY DISPENSE BEADS, POWDERS INTO MICROPLATES AND TUBES

- eCONOMICAL VS PRICEY PRE-FILLED LYSIS TUBES

- ACCURATE, FAST, SIMPLE, CUSTOMIzABLE

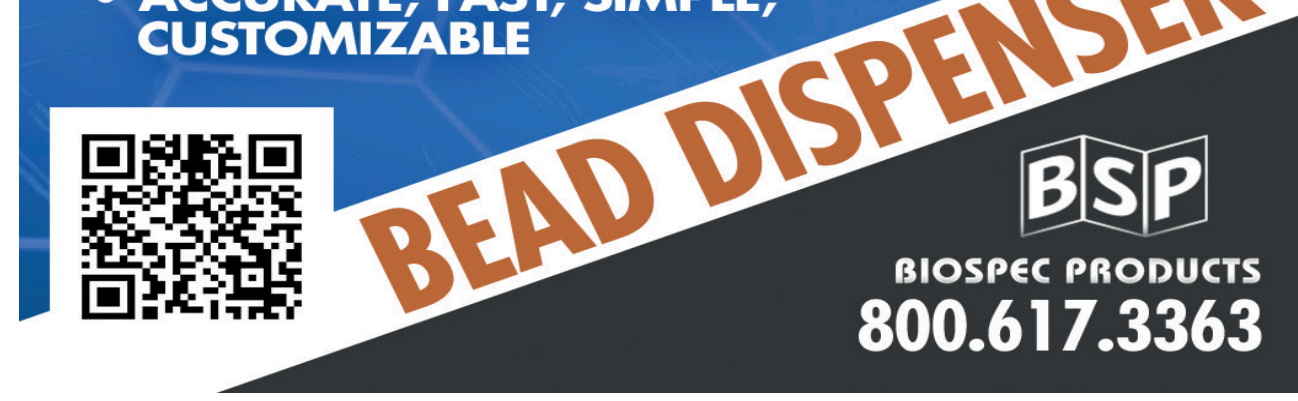

